Document downloaded from:

http://hdl.handle.net/10251/60216

This paper must be cited as:

Llorens, L.; Llorens Molina, JA.; Agnello, S.; Boira Tortajada, H. (2014). Geographical and environment-related variations of essential oils in isolated populations of Thymus richardii Pers. in the Mediterranean basin. Biochemical Systematics and Ecology. 56:246-254. doi:10.1016/j.bse.2014.05.007.

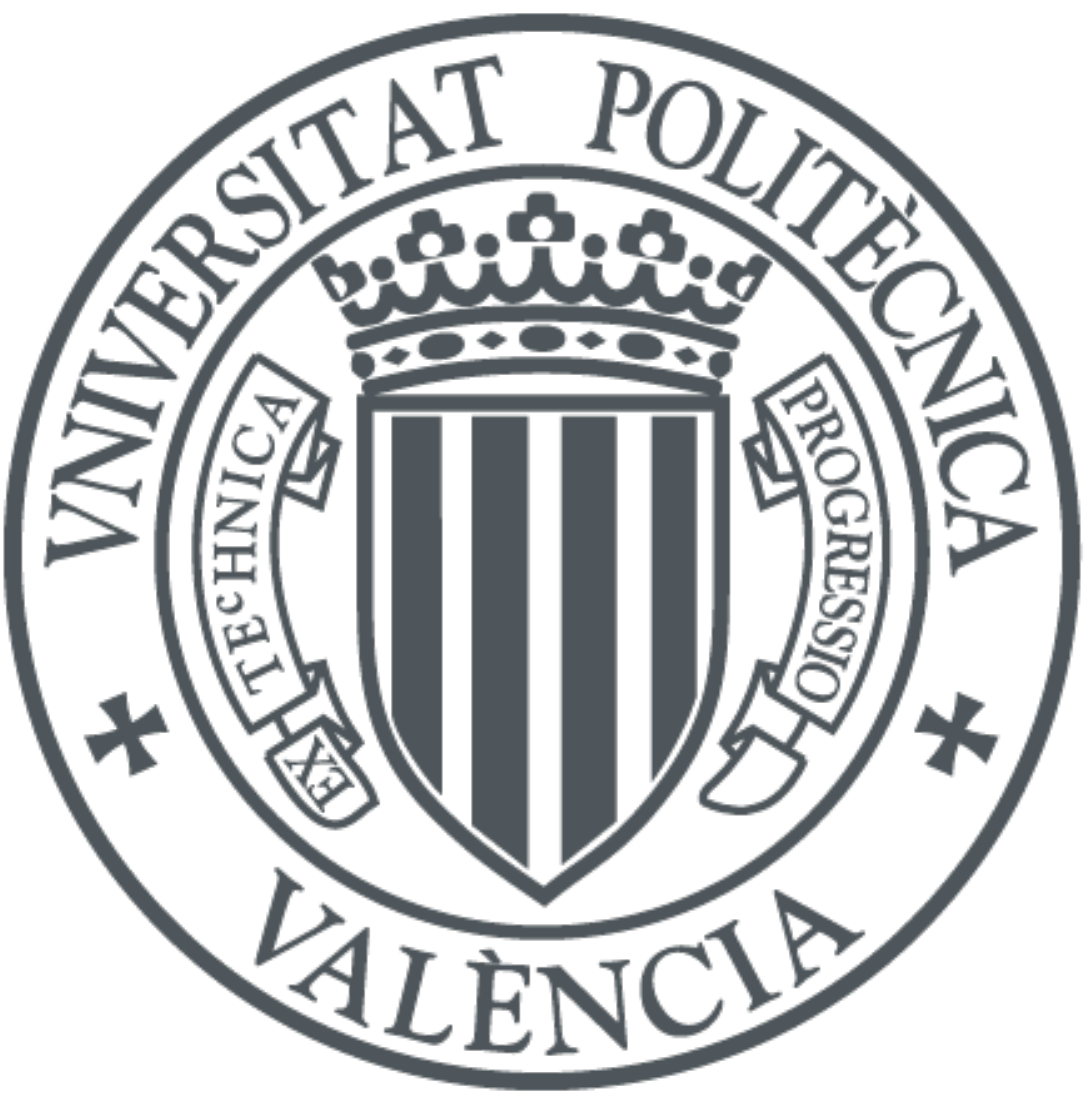

The final publication is available at

http://dx.doi.org/10.1016/j.bse.2014.05.007

Copyright Elsevier

Additional Information 


\title{
Geographical and environment-related variations of essential oils in isolated populations of Thymus richardii Pers. in the Mediterranean basin
}

\author{
L. Llorens ${ }^{\mathrm{a},}{ }^{*}$, J.A. Llorens-Molina ${ }^{\mathrm{b}, 1}$, S. Agnello ${ }^{\mathrm{c}, 2}$, H. Boira ${ }^{\mathrm{b}, 3}$ \\ a Laboratori de Botànica, Departament de Biologia, University of Balearic Island (UIB), Carretera de Valdemossa km. 7,5, 07122 Palma de \\ Mallorca, Spain \\ ${ }^{\mathrm{b}}$ Departamento de Ecosistemas Agroforestales, IAM-UPV, Camino de Vera s/n, 46022 Valencia, Spain

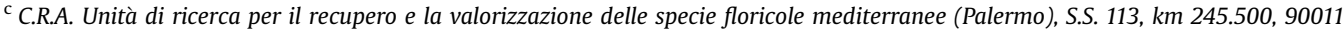 \\ Bagheria, Italy
}

\section{A R T I C L E I N F O}

\section{Article history:}

Received 27 October 2013

Accepted 24 May 2014

Available online

\section{Keywords:}

Thymus richardii

Essential oils

Chemotypes

Environmental factors

\begin{abstract}
A B S T R A C T
Composition of essential oils of different populations of Thymus richardii grex of six localities from Bosnia-Herzegovina (Konjic, Borci), Spain (Majorca, Ibiza, Valencia) and Italy (Marettimo, Sicily) were determined by GC/FID and GC/MS. The main constituents in most of the samples were aromatic monoterpenes corresponding to non-phenolic cyclic compounds ( $\mathrm{p}$-cymene, $\gamma$-terpinene). The highest monoterpene concentrations were found in the Bosnian samples (70\%), and the lowest in samples from the Balearic Islands $(<30 \%$; Ibiza and Majorca). Sesquiterpenes were the major component (average $>50 \%$ ) in samples from Majorca with $\beta$-bisabolene $(>40 \%)$ being the principal constituent. Discriminant analysis (LDA) shows the differentiation of two chemotypes: A (phenol chemotype), with p-cymene and $\gamma$-terpinene as characteristic compounds and $B$, with $\beta$-bisabolene and carvacrol, as major and significative compounds. The occurrence of the chemotypes was related to summer positive precipitation and to deep of soils.
\end{abstract}

(C) 2014 Elsevier Ltd. All rights reserved.

\section{Introduction}

The plant family Lamiaceae is common in the Mediterranean region, with members exhibiting highly diversified ecologies. Species in the family constitute an important component of Mediterranean shrub vegetation, especially in dry and arid environments. Secondary metabolites produced by these plants play important ecological and biological roles. The diversity of biological activities of the essential oils of the Lamiaceae may be a consequence of their rich chemical diversity, and in part explains the large number of studies investigating the chemical composition of plants in this family, and its relationship to

\footnotetext{
* Corresponding author. Tel.: +34 61736638; fax: +34 971173184.

E-mail addresses: lleonard.llorens@uib.es (L. Llorens), juallom2@qim.upv.es (J.A. Llorens-Molina), santo.agnello@entecra.it (S. Agnello), hboira@eaf.upv. es (H. Boira)

1 Tel.: +34620248375

2 Tel.: +39091 909090; fax: +39091909089.

3 Tel.: +34 676832096 .
} 
fields including taxonomy, biogeography and medicine (for example, see Sáez and Stahl-Biskup, 2002a; Giuliani and MaleciBini, 2008; Jassbi et al., 2012; Lakusic et al., 2012) and citations.

One of the most important genera of the Lamiaceae in the Mediterranean region is the genus Thymus Benth., which includes approximately 110 widespread species (Morales, 1997). Thymus richardii Pers. (included in the section Serpyllum, subsection Insulares, -Jalas, 1971-), is an example of a species having a relictual, fragmented and scattered distribution under. It was first described in Majorca (Balearic Islands) by Persoon (1806), and later from Sicily (Gussone, 1843), Ibiza (Font Quer, 1935), Valencia (Bolòs and Vigo, 1983), and Bosnia-Herzegovina (Jalas, 1971, 1972).

Based on morphological characteristics (occasionally determined using herbarium material), the Thymus taxon constitute an aggregation of four infraspecific taxa (Jalas, 1971, 1972) distributed in few localities, where they generally form sparse and isolated populations comprising few plants, including T. richardii subsp. richardii (Majorca, Spain; Bosnia-Herzegovina), $T$. richardii subsp. ebusitanus (Font Quer) Jalas (Ibiza, Spain), T. richardii subsp. nitidus (Guss.) Jalas (Marettimo Island, Sicily, Italy) and T. richardii subsp. vigoi Riera, Güemes \& Rosselló (Valencia, Spain). However, in various studies some of these have been treated as differentiated species, including Thymus nitidus Guss. (= T. richardii subsp. nitidus) and Thymus aureopunctatus Beck (= T. richardii subsp. richardii, Bosnio-Herzegovinian populations).

From a phytosociological point of view the Bosnian populations form part of the Illyrian black pine woods (Orno-ericenion "dolomiticum"), and the subspecies also occurs in xeric stands of oak (Konjic) and thermophilous beech woods (Borci). The island of Marettimo (Sicily) is composed entirely of white and grey dolomite. Similar edaphic features occur in all the populations of Ibiza. The populations at these two locations have a subrupiculous character, or grow on litosols.

Another feature is that in these two localities the plants are sometimes integrated into the Mediterranean scrubland vegetation (Rosmarinetalia). In Valencia (La Safor) the plants only grow in this type of vegetation, which substitutes for Pinus halepensis or Quercus ilex forest. However, in Majorca the populations (Puig Major) are exclusively found in rocky crevices on the tops of mountains on the island, and have a clear rupiculous character.

The essential oils of Thymus species have been the subject of numerous studies (for example, see Sáez and Stahl-Biskup, 2002b; Stahl-Biskup, 2002 and citation). Some studies have investigated the terpene composition and its variability in relation to edaphic (Martonfi et al., 1994), environmental (Boira and Blanquer, 1998), altitudinal (Delazar et al., 2011), and seasonal and cultural (Letchamo and Gosselin, 1995; Aziz et al., 2008; Sharafzadeh et al., 2011) conditions. However, few studies have considered chemotaxonomic (Tzakou and Constantinidis, 2005) or biogeographic aspects (Blanco et al., 2012).

Wild Thymus species are highly chemically polymorphic, with variability in the essential oil composition being related to various environmental and genetic factors (Thompson, 2002; Loziene and Venskutonis, 2005; Pluhar et al., 2007).

Most of the volatile terpenoids in Thymus oils belong to the monoterpene group, which usually comprise more than 90\% of the total. Sesquiterpenes are always present but, with only few exceptions, as a minor component (Stahl-Biskup, 2002). For example, Thymus samius Ronniger (Tzakou and Constantinidis, 2005), determinate varieties of Thymus serpyllum (Miller) Benth. (Paaver et al., 2008) and T. richardii Pers. subsp. nitidus (Guss.) Jalas (Bader et al., 2001) contain (E)-nerodiol, (E)caryophyllene, germacrene $\mathrm{D}$ and (very rarely) $\beta$-bisabolene as major sesquiterpenes.

Moreover, it seems that some species having phenol chemotypes (Thymus hyemalis Lange, Thymus mastichina L., Thymus zygis L. and Thymus piperella L.) correspond to archaic forms with the original endemic distribution, while species having geraniol are more evolved and have a more northern geographic distribution. This is consistent with previous studies reporting that the abundance of phenolic compounds is associated with typical Mediterranean climatic and edaphic conditions, and that the non-phenolic chemotypes among Thymus species must overcome environmental conditions that differ from the above (e.g. cold winters) (Bruneton, 1995).

In defining chemotypes the biosynthetic precursors of phenolic compounds ( $\gamma$-terpinene and $p$-cymene) are commonly taken into account. Correlations between the amounts of phenolic compounds and their biosynthetic precursors tend to confirm the traditional pathway in which p-cymene is the immediate precursor of thymol and carvacrol (Thompson et al., 2003). More recently it has been proposed that the formation of thymol and carvacrol is catalyzed by single P450s directly from $\gamma$-terpinene via a two-step oxidation, whereas p-cymene is a side product resulting from premature release of the substrate from the enzyme active site (Crocoll et al., 2010). In relation to the phenolic nature of the thyme taxa, both possibilities lead to the consideration of thymol and carvacrol with their derivatives and precursors as a whole.

Knowledge of the terpene composition of the T. richardii group is restricted to two taxa: T. richardii subsp. nitidus (Bader et al., 2001) and T. aureopunctatus (Cavara et al., 2009). The former is characterized by being rich in $\beta$-bisabolene (32.3\%), while the second is a thymol chemotype (34.5\% thymol).

In the present study grex of taxa integrated into T. richardii were studied with the following objectives: 1) to determine the terpene composition of the essential oils among the various populations studied; 2 ) to identify discriminating compounds to differentiate chemical groups; and 3) to assess the relationship between chemical composition and biogeography, as well as climatic and soil parameters.

\section{Material and methods}

\subsection{Plant material}

During summer 2011, samples of wild populations of the T. richardii group were collected in situ from their place of origin (Fig. 1; Table 1). Sampled plants were in the full flowering stage, and were collected during in the sunniest time of the day. For 


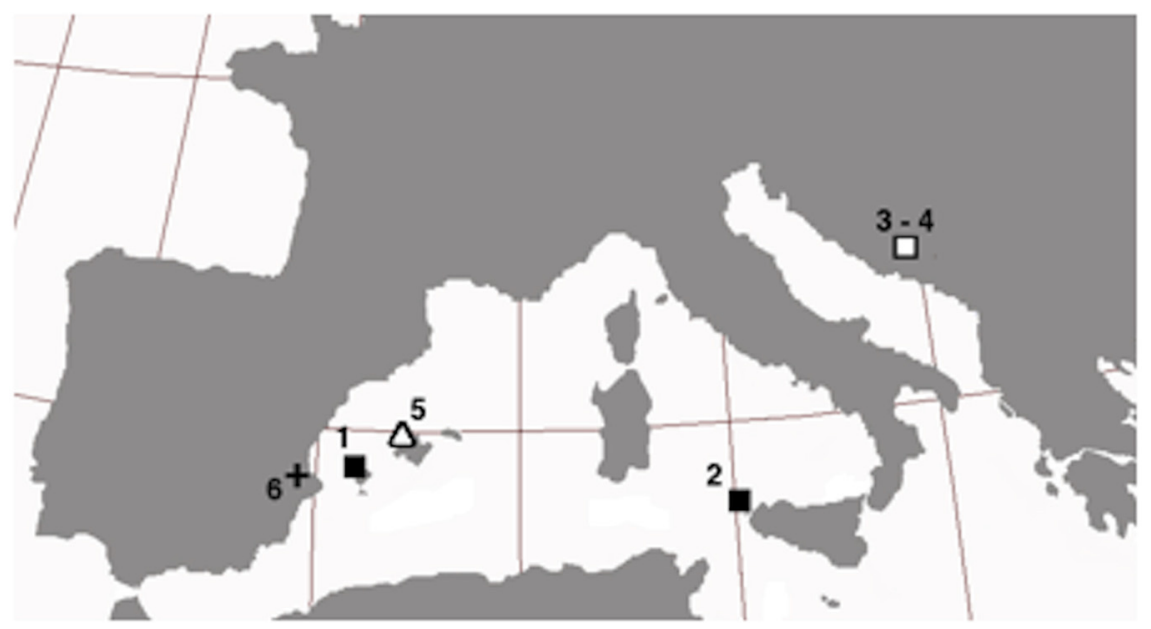

Fig. 1. Map of the western Mediterranean basin showing the geographical distribution of the Thymus richardii populations analyzed. Numbers 1-5: population codes. Symbols indicate bioclimates ( $\square$ : temperate humid; $\Delta$ supra-Mediterranean humid; +: thermo-Mediterranean humid/subhumid; $\mathbf{\square}$ : thermoMediterranean dry).

each population three samples by plant from ten individuals were collected randomly. The sampled plants were $>10 \mathrm{~m}$ apart, to avoid sampling from the same parent. Branches with leaves were collected from each individual and stored at $-40{ }^{\circ} \mathrm{C}$ until extracted for volatile fraction analysis. The woody parts were separated from the leaves, young branches and inflorescences.

\subsection{Climate data and bioclimatic index}

Climate data were obtained from the meteorological station nearest to each study area. The data covered a period of 30 years for the Safor (Valencia) and Marettimo (Favignana) areas, and 20 years for the Konjic, Borci (Bosnia), Ibiza and Majorca areas.

To investigate relationships between climatic factors and the composition of essential oils we used several bioclimatic indices (Rivas-Martínez et al. 2011) and the Giacobbe index (IG) (Giacobbe, 1938, 1959). The bioclimatic parameters investigated are mostly related to summer, as this is the critical period for Mediterranean flora, and included: ETP, potential evapotranspiration; Ic, continentality index; It, thermicity index; Io, ombrothermic index; IG, summer drought index; PPv, summer precipitation for the three consecutive warmest months in the year; TPv, the sum of the monthly average temperatures for the three consecutive warmest months (Table 2).

\subsection{Essential oil extraction and analysis}

Simultaneous distillation-extraction (SDE) was performed for $2 \mathrm{~h}$ using a Likens and Nickerson device with dichloromethane as the organic solvent. The extracts were dried with anhydrous sodium sulfate and concentrated to a $2 \mathrm{~mL}$ volume

\section{Table 1}

Geobotanical data for the studied populations of T. richardii. UTM: UTM coordinates; $\left({ }^{*}\right)$ : TMed $=$ ThermoMediterranean; MMed = MesoMediterranean; SMed $=$ SupraMediterranean; Temp $=$ temperate.

\begin{tabular}{|c|c|c|c|c|c|}
\hline Taxa $\left({ }^{*}\right)$ & Locality (UTM) & Altitude (m) & Bioclimate $\left({ }^{*}\right)$ & Community & Habitat \\
\hline $\begin{array}{l}\text { 1- Thymus richardii } \\
\text { subsp. ebusitanus }\end{array}$ & Ibiza 31S 35E/43N & $160-280$ & TMed dry & $\begin{array}{l}\text { Brassico balearicae-Helichrysion } \\
\text { rupestris (Asplenietea trichomanis) }\end{array}$ & $\begin{array}{l}\text { Subrupiculous. Limestone } \\
\text { rock crevices with } \\
\text { chasmophytic vegetation. } \\
\text { Sea breeze affected }\end{array}$ \\
\hline $\begin{array}{l}\text { 2- Thymus richardii } \\
\text { subsp. nitidus }\end{array}$ & $\begin{array}{l}\text { Sicily/Marettimo } \\
33 \mathrm{~S} 24 \mathrm{E} / 42 \mathrm{~N}\end{array}$ & $160-450$ & TMed dry & $\begin{array}{l}\text { Dianthion rupicolae (Asplenietea } \\
\text { trichomanis) }\end{array}$ & $\begin{array}{l}\text { Rupiculous. Limestone } \\
\text { rock crevices with } \\
\text { chasmophytic vegetation. } \\
\text { Sea breeze affected }\end{array}$ \\
\hline $\begin{array}{l}\text { 3- Thymus richardii } \\
\text { subsp. richardii }\end{array}$ & $\begin{array}{l}\text { Bosnia } 1 / \text { Konjic-Spiljani } \\
34 \mathrm{~T} 25 \mathrm{E} / 48 \mathrm{~N}\end{array}$ & 350 & $\begin{array}{l}\text { Temp/SMed } \\
\text { humid }\end{array}$ & $\begin{array}{l}\text { Peucedanion neumayeri } \\
\text { (Thero-Brachypodietea) }\end{array}$ & Slopes \\
\hline $\begin{array}{l}\text { 4- Thymus richardii } \\
\text { subsp. richardii }\end{array}$ & $\begin{array}{l}\text { Bosnia 2/Borci-Bjelasnica } \\
34 \mathrm{~T} 25 \mathrm{E} / 48 \mathrm{~N}\end{array}$ & 800 & Temp humid & $\begin{array}{l}\text { Peucedanion neumayeri } \\
\text { (Thero-Brachypodietea) }\end{array}$ & Slopes. Dolomitophyte \\
\hline $\begin{array}{l}\text { 5- Thymus richardii } \\
\text { subsp. richardii }\end{array}$ & $\begin{array}{l}\text { Majorca/Puig Major } 31 \mathrm{~S} \\
48 \mathrm{E} / 44 \mathrm{~N}\end{array}$ & $1100-1300$ & $\begin{array}{l}\text { SMed/MMed } \\
\text { humid }\end{array}$ & $\begin{array}{l}\text { Brassico balearicae-Helichrysion } \\
\text { rupestris (Asplenietea trichomanis) }\end{array}$ & $\begin{array}{l}\text { Rupiculous. Limestone } \\
\text { rock crevices with } \\
\text { chasmophytic vegetation }\end{array}$ \\
\hline $\begin{array}{l}\text { 6- Thymus richardii } \\
\text { subsp. vigoi }\end{array}$ & $\begin{array}{l}\text { Valencia/La Safor 30S } \\
73 \mathrm{E} / 43 \mathrm{~N}\end{array}$ & $550-650$ & $\begin{array}{l}\text { MMed/TMed } \\
\text { humid }\end{array}$ & $\begin{array}{l}\text { Rosmarinion officinalis } \\
\text { (Rosmarinetea officinalis) }\end{array}$ & Slopes. Dolomitophyte \\
\hline
\end{tabular}

$\left({ }^{*}\right)$ Vouchers specimen collected in the studied populations has been included in the Herbarium of Politechnic University of Valencia (VALA, $\mathrm{n}^{\circ}$ 7245-7251). Syntaxonomical nomenclature according to Ritter-Studnička, 1967 and Rivas-Martínez et al., 2001. 
Table 2

Bioclimatic indices of $T$. richardii areas in the Mediterranean basin.

\begin{tabular}{|c|c|c|c|c|c|c|c|}
\hline Locality & $\left.\mathrm{It}^{\mathrm{a}}\right)$ & $\left.\mathrm{Ic}^{\mathrm{b}}\right)$ & $\left.\mathrm{Io}^{\mathrm{c}}\right)$ & $\left.P p v^{d}\right)$ & $\left.\mathrm{Ttv}^{\mathrm{e}}\right)$ & $\mathrm{ETP}^{\mathrm{f}}$ ) & $\left.\mathrm{IG}^{\mathrm{g}}\right)$ \\
\hline Bosnia 1 & 308.50 & 9.78 & 7.16 & 273.00 & 574.50 & 208.96 & 13.68 \\
\hline Bosnia 2 & 373.75 & 11.92 & 5.35 & 242.90 & 665.00 & 204.10 & 10.56 \\
\hline Valencia & 518.17 & 12.11 & 2.76 & 50.90 & 717.00 & 289.88 & 2.04 \\
\hline Sicily & 526.25 & 8.75 & 1.78 & 16.00 & 715.00 & 295.34 & 0.64 \\
\hline Ibiza & 507.83 & 10.09 & 1.90 & 44.40 & 730.00 & 304.82 & 1.72 \\
\hline Majorca & 272.92 & 7.53 & 9.52 & 83.00 & 482.00 & 103.24 & 4.80 \\
\hline
\end{tabular}

$\mathrm{T}$ : yearly average temperature. $\mathrm{m}$ : average temperature of the minima of the coldest month of the year. M: average temperature of the maxima of the coldest month of the year. T max: average temperature of the warmest month. T min: average temperature of the coldest month. Tm: average temperature of the each month. P: total yearly precipitation.

a It: Thermicity index, $10^{*}(\mathrm{~T}+\mathrm{M}+\mathrm{m})$.

b Ic: Continentality index, $\mathrm{T}$ max $-\mathrm{T}$ min.

c Io: Ombrothermic index, $(\mathrm{P} / 12)^{*} 10 / \sum \mathrm{Tm}$.

d Ppv: Summer precipitation in mm of the three consecutive warmest months in the year.

e Ttv: Value in tenths of degree resulting from the sum of the monthly average temperatures of the three consecutive warmest months in the year.

f ETP: Potential evapotranspiration (Thornthwaite).

g IG (Giacobbe index): (Pjun + Pjul + Paug)/Tª of the warmest month.

using a Buchi R-3000 rotary evaporator equipped with a vacuum pump (Buchi V-700) combined with a vacuum module (Buchi V-801 Easy Vac) (Chaintreau, 2001).

Analysis of the SDE extracts was carried out using GC and GC-MS. A Clarus 500 GC (PerkinElmer Inc., Wellesley, MA, EEUU) chromatograph equipped with a FID detector and a ZB-5 capillary column ( $30 \mathrm{~m} \times 0.25 \mathrm{~mm}$ i.d. $\times 0.25 \mu \mathrm{m}$; Phenomenex Inc., Torrance, CA, EEUU) was used for quantitative analyses (injection volume $1 \mu \mathrm{L}$ ). The GC oven temperature program was from $50{ }^{\circ} \mathrm{C}$ to $250{ }^{\circ} \mathrm{C}$ at a rate of $3{ }^{\circ} \mathrm{C} \mathrm{min}^{-1}$. Helium was used as the carrier gas $\left(1.2 \mathrm{~mL} \mathrm{~min}^{-1}\right)$, and the injector and detector temperatures were set at $250{ }^{\circ} \mathrm{C}$. The percentage composition of the essential oil was computed from the GC peak areas (without correction factors) using Total Chrom 6.2 (Perkin Elmer Inc.) software.

Analysis by GC-MS was performed using the same capillary column, mobile phase and operating conditions as described above for the GC analysis. The ionization source temperature was $200{ }^{\circ} \mathrm{C}$, and a $70 \mathrm{eV}$ electron impact mode was used. MS spectra were obtained using the total ion scan (TIC) mode (mass range $m / z 45-500$ uma). The total ion chromatograms and mass spectra were processed using Turbomass 5.4 (Perkin Elmer Inc.) software.

The extract components were identified based on their Kovats retention indexes relative to C7-C30 Saturated Alkanes $\left(\right.$ Sigma-Aldrich ${ }^{\circledR}$ ), computer matching with the NIST MS Search 2.0 library, and comparison of their mass spectra with those of previously published data.

\subsection{Data processing}

The percentage compositions of the oil samples were used as matrix elements to determine the relationship between the 18 samples from the six studied populations.

The data were analyzed using the Statgraphics Centurion V. 06 statistical package. The matrix of compounds was analyzed using principal component analysis (PCA) and discriminant function analysis to assess the heterogeneity of populations and potential chemotypes. Canonical correspondence analysis (CCA) was applied to investigate relationships between principal compounds and population and ecological factors, using the Multi-Variate Statistical Package (MVSP) V 3.01.

\section{Results and discussion}

\subsection{Essential oil content}

A total of 72 compounds were identified, accounting for $91-99 \%$ of the volatile constituents. The composition of the essential oil (arranged in Table 3 in the order of elution) was dominated by the monoterpene fraction, as has been reported for other Thymus species (Stahl-Biskup, 2002; Skoula and Harborne, 2002), except for those populations in which $\beta$-bisabolene was one of the major compounds.

The main constituents in most of the samples were aromatic monoterpenes (average concentration $>40 \%$ ), corresponding to non-phenolic cyclic compounds ( $p$-cymene, $\gamma$-terpinene). The highest monoterpene concentrations were found in the Bosnio-Herzegovinian samples (70\%; Konjic and Borci), and the lowest in samples from the Balearic Islands (<30\%; Ibiza and Majorca). Related to these compounds, a second important group of phenolic cyclic compounds (thymol and methyl thymol ether) reached average levels of $2-15 \%$.

Sesquiterpenes were the major component (average $>50 \%$ ) in samples from Majorca (Fig. 2), with $\beta$-bisabolene ( $>40 \%$ ), $\beta$ caryophyllene and caryophyllene oxide being the principal constituents. The presence of $\beta$-bisabolene in samples from Marettimo and Ibiza (17 and 12\%, respectively) differentiated these populations from the Valencia (Safor), Bosnia 1 (KonjicSpiljani) and Bosnia 2 (Borci-Bjelasnica) populations. 
Table 3

Chemical Composition [\%] of Essential Oils of Thymus richardii samples from 18 Mediterranean populations.

\begin{tabular}{|c|c|c|c|c|c|c|c|c|c|c|c|c|c|c|c|c|c|c|c|c|}
\hline \multirow[t]{2}{*}{ Localities } & \multirow[b]{3}{*}{$\left.K I E^{\mathrm{a}}\right)$} & \multirow[b]{3}{*}{$\left.K I L^{\mathrm{b}}\right)$} & \multirow{2}{*}{\multicolumn{3}{|c|}{$\begin{array}{l}\text { Bosnia } 1 \\
\text { (Konnic/ } \\
\text { Spiljani) }\end{array}$}} & & & & Sicily & & & Ibiza & & & Valen & cia & & Majoı & & \\
\hline & & & & & & $\begin{array}{l}\text { Borc } \\
\text { Bjelas }\end{array}$ & snica) & & 115 & & & Island & & & all & & & & & \\
\hline Compounds & & & 1 & 2 & 3 & 4 & 5 & 6 & 7 & 8 & 9 & 10 & 11 & 12 & 13 & 14 & 15 & 16 & 17 & 18 \\
\hline$\alpha$-Thujene & 929 & 930 & 1.0 & 0.8 & 0.9 & 0.6 & 1.0 & 0.9 & 0.7 & 0.8 & 0.7 & 0.3 & 0.0 & 0.3 & 0.8 & 0.9 & 0.7 & 0.0 & 0.0 & 0.0 \\
\hline$\alpha$-Pinene & 937 & 939 & 1.0 & 1.0 & 1.0 & 0.4 & 0.7 & 1.2 & 1.5 & 1.3 & 1.2 & 0.8 & 0.7 & 2.2 & 0.9 & 1.5 & 1.3 & 0.0 & 0.0 & 0.0 \\
\hline Camphene & 953 & 954 & 1.3 & 1.8 & 1.5 & 0.4 & 0.6 & 2.1 & 0.0 & 0.0 & 0.0 & 0.0 & 0.0 & 0.1 & 0.3 & 0.7 & 1.6 & 0.0 & 0.0 & 0.0 \\
\hline Sabinene & 975 & 975 & 0.0 & 0.0 & 0.0 & 0.0 & 0.1 & 0.1 & 0.1 & 0.0 & 0.0 & 0.1 & 0.0 & 0.2 & 0.5 & 0.3 & 0.3 & 0.0 & 0.0 & 0.0 \\
\hline 1-Octen-3-ol & 983 & 979 & 0.2 & 0.2 & 0.2 & 0.0 & 0.2 & 0.3 & 0.1 & 0.2 & 0.0 & 0.2 & 0.0 & 0.2 & 0.3 & 0.3 & 0.5 & 0.0 & 0.0 & 0.0 \\
\hline 3-Octanone & 988 & 984 & 0.8 & 1.4 & 0.7 & 2.4 & 3.2 & 1.4 & 0.0 & 0.0 & 0.0 & 0.8 & 0.3 & 0.6 & 0.1 & 0.4 & 0.0 & 0.0 & 0.0 & 0.0 \\
\hline$\beta$-Myrcene & 990 & 991 & 0.2 & 0.3 & 0.2 & 3.1 & 4.2 & 0.5 & 0.4 & 0.1 & 0.0 & 0.9 & 0.4 & 1.5 & 0.8 & 0.6 & 0.5 & 0.3 & 0.0 & 0.2 \\
\hline 3-Octanol & 993 & 991 & 0.0 & 0.0 & 0.0 & 0.0 & 0.0 & 0.0 & 2.1 & 0.8 & 0.6 & 0.0 & 0.0 & 0.0 & 0.0 & 0.0 & 0.0 & 0.0 & 0.0 & 0.0 \\
\hline$\alpha$-Phellandrene & 1006 & 1003 & 0.0 & 0.0 & 0.0 & 2.0 & 2.8 & 0.0 & 0.0 & 0.0 & 0.0 & 0.0 & 0.0 & 0.0 & 0.0 & 0.0 & 0.0 & 0.0 & 0.0 & 0.0 \\
\hline Sylvestrene $<i s o>$ & 1011 & 1009 & 0.0 & 0.0 & 0.0 & 0.0 & 0.0 & 0.0 & 0.0 & 0.0 & 0.0 & 0.2 & 0.0 & 0.6 & 0.0 & 0.0 & 0.0 & 0.0 & 0.0 & 0.0 \\
\hline$\alpha$-Terpinene & 1018 & 1017 & 0.2 & 0.2 & 0.2 & 0.2 & 0.5 & 0.4 & 0.4 & 0.7 & 0.4 & 0.7 & 0.2 & 0.5 & 0.6 & 0.4 & 0.2 & 0.0 & 0.0 & 0.1 \\
\hline p-Cymene & 1028 & 1025 & 60.0 & 58.4 & 67.7 & 35.0 & 36.4 & 57.3 & 15.3 & 25.1 & 20.4 & 15.1 & 7.0 & 18.6 & 18.1 & 36.2 & 48.2 & 1.0 & 0.0 & 0.2 \\
\hline Limonene & 1032 & 1029 & 0.3 & 0.3 & 0.4 & 0.0 & 0.8 & 0.4 & 16.7 & 8.8 & 13.0 & 1.6 & 0.5 & 1.3 & 4.9 & 4.4 & 0.6 & 0.0 & 0.0 & 0.0 \\
\hline 1,8-Cineole & 1035 & 1031 & 0.0 & 0.0 & 0.0 & 0.0 & 0.0 & 0.0 & 0.0 & 0.0 & 0.0 & 1.6 & 0.6 & 1.5 & 2.1 & 1.5 & 3.4 & 0.0 & 0.0 & 0.0 \\
\hline Benzene acetaldehyde & 1045 & 1042 & 0.1 & 0.0 & 0.0 & 0.0 & 0.2 & 0.0 & 0.0 & 0.4 & 0.0 & 0.0 & 0.0 & 0.0 & 0.0 & 0.0 & 0.0 & 0.0 & 0.0 & 0.6 \\
\hline$\gamma$-Terpinene & 1061 & 1060 & 5.3 & 5.2 & 3.5 & 4.3 & 3.4 & 6.6 & 2.8 & 7.0 & 4.4 & 6.7 & 2.3 & 4.0 & 17.6 & 10.2 & 11.5 & 0.3 & 0.0 & 0.2 \\
\hline (Z)-Sabinene hydrate & 1072 & 1070 & 0.6 & 0.4 & 0.4 & 0.8 & 0.5 & 0.5 & 0.9 & 1.2 & 0.7 & 0.0 & 0.0 & 0.0 & 10.4 & 5.1 & 2.2 & 0.0 & 0.0 & 0.0 \\
\hline Terpinolene & 1090 & 1086 & 0.0 & 0.0 & 0.0 & 0.0 & 0.0 & 0.0 & 0.0 & 0.0 & 0.0 & 0.0 & 0.0 & 0.4 & 0.0 & 0.0 & 0.0 & 0.0 & 0.0 & 0.0 \\
\hline Linalool & 1097 & 1097 & 0.1 & 0.0 & 0.0 & 0.0 & 0.0 & 0.0 & 2.2 & 1.7 & 1.0 & 0.0 & 0.0 & 0.2 & 1.7 & 1.1 & 4.8 & 0.2 & 0.1 & 0.4 \\
\hline (E)-Sabinene hydrate & 1102 & 1098 & 0.2 & 0.1 & 0.2 & 0.1 & 0.4 & 0.2 & 0.0 & 0.0 & 0.0 & 0.0 & 0.0 & 0.0 & 0.0 & 0.0 & 0.0 & 0.0 & 0.0 & 0.0 \\
\hline (E)-Limonene oxyde & 1138 & 1137 & 0.0 & 0.0 & 0.0 & 0.0 & 0.0 & 0.0 & 0.0 & 0.0 & 0.0 & 0.0 & 0.0 & 0.0 & 0.0 & 0.0 & 0.0 & 0.0 & 0.0 & 0.0 \\
\hline Camphor & 1150 & 1146 & 0.3 & 0.3 & 0.2 & 0.1 & 0.2 & 1.0 & 0.0 & 0.9 & 0.0 & 0.0 & 0.0 & 0.0 & 0.0 & 0.0 & 0.0 & 0.0 & 0.1 & 0.1 \\
\hline 1-Terpinen-4-ol & 1182 & 1177 & 1.2 & 2.6 & 2.3 & 1.6 & 0.9 & 2.0 & 0.6 & 0.4 & 0.3 & 0.1 & 0.0 & 0.0 & 0.7 & 1.5 & 4.0 & 0.3 & 0.4 & 0.4 \\
\hline p-Cymen-8-ol & 1188 & 1183 & 0.3 & 0.3 & 0.3 & 0.2 & 0.4 & 0.3 & 0.0 & 0.0 & 0.0 & 0.9 & 0.7 & 0.8 & 1.2 & 0.9 & 1.0 & 0.0 & 0.0 & 0.0 \\
\hline$\alpha$-Terpineol & 1193 & 1189 & 0.6 & 0.6 & 0.5 & 0.5 & 0.5 & 0.5 & 0.0 & 0.0 & 0.0 & 0.3 & 0.1 & 0.4 & 0.0 & 0.1 & 0.1 & 0.0 & 0.0 & 0.0 \\
\hline (Z)-Dihydrocarvone & 1195 & 1193 & 0.0 & 0.0 & 0.0 & 0.0 & 0.0 & 0.0 & 0.6 & 0.0 & 0.1 & 0.2 & 0.1 & 0.2 & 0.0 & 0.0 & 0.0 & 0.0 & 0.0 & 0.0 \\
\hline (E)-Dihydrocarvone & 1202 & 1201 & 0.0 & 0.0 & 0.0 & 0.0 & 0.0 & 0.0 & 5.7 & 0.0 & 2.5 & 0.2 & 0.0 & 0.1 & 0.1 & 0.1 & 0.2 & 0.0 & 0.0 & 0.3 \\
\hline (E)-Carveol & 1218 & 1217 & 0.0 & 0.0 & 0.0 & 0.0 & 0.0 & 0.0 & 0.7 & 0.0 & 0.0 & 0.5 & 0.4 & 0.5 & 0.0 & 0.0 & 0.0 & 0.0 & 0.0 & 0.0 \\
\hline Methyl thymol & 1232 & 1232 & 16.8 & 14.1 & 10.5 & 13.0 & 9.6 & 13.8 & 8.8 & 2.4 & 14.9 & 0.0 & 0.0 & 0.0 & 0.0 & 0.0 & 0.0 & 8.0 & 10.9 & 10.3 \\
\hline Methyl carvacrol & 1246 & 1240 & 0.0 & 1.0 & 0.1 & 3.5 & 3.8 & 0.1 & 0.0 & 0.0 & 0.0 & 13.9 & 18.9 & 14.6 & 0.0 & 0.0 & 0.0 & 0.5 & 0.1 & 1.7 \\
\hline Thymoquinone & 1256 & 1252 & 0.1 & 0.0 & 0.0 & 0.1 & 0.3 & 0.0 & 0.4 & 0.1 & 0.1 & 0.0 & 0.0 & 0.1 & 0.3 & 0.9 & 0.0 & 0.0 & 0.0 & 0.0 \\
\hline Bornyl acetate & 1291 & 1289 & 0.4 & 0.8 & 0.2 & 0.0 & 0.0 & 0.6 & 0.0 & 0.0 & 0.0 & 0.0 & 0.0 & 0.0 & 0.0 & 0.0 & 0.0 & 0.0 & 0.0 & 0.0 \\
\hline Thymol & 1294 & 1290 & 4.0 & 3.9 & 2.8 & 21.6 & 21.8 & 3.8 & 1.5 & 13.9 & 2.8 & 14.7 & 6.5 & 4.9 & 18.1 & 12.8 & 1.5 & 11.4 & 9.0 & 6.2 \\
\hline Carvacrol & 1305 & 1299 & 0.1 & 0.0 & 0.4 & 0.2 & 0.5 & 0.3 & 15.2 & 0.0 & 8.0 & 12.8 & 10.8 & 9.8 & 0.1 & 5.2 & 0.0 & 6.4 & 10.5 & 5.2 \\
\hline Thymol acetate & 1356 & 1352 & 0.1 & 0.0 & 0.0 & 0.0 & 0.0 & 0.0 & 0.0 & 0.0 & 0.0 & 0.0 & 0.0 & 0.1 & 0.0 & 0.0 & 0.0 & 0.0 & 0.0 & 0.0 \\
\hline$\alpha$-Copaene & 1380 & 1377 & 0.0 & 0.0 & 0.0 & 0.0 & 0.0 & 0.0 & 0.0 & 0.0 & 0.0 & 0.1 & 0.5 & 0.3 & 0.0 & 0.0 & 0.0 & 0.0 & 0.0 & 0.0 \\
\hline$\beta$-Bourbonene & 1387 & 1388 & 0.0 & 0.0 & 0.0 & 0.0 & 0.0 & 0.0 & 0.0 & 0.8 & 0.0 & 0.1 & 0.0 & 0.0 & 0.0 & 0.2 & 0.5 & 0.4 & 0.3 & 1.0 \\
\hline$\beta$-Caryophyllene & 1424 & 1419 & 1.8 & 2.7 & 2.3 & 4.0 & 1.9 & 2.5 & 0.4 & 2.6 & 1.1 & 6.1 & 12.0 & 10.2 & 4.8 & 3.2 & 4.5 & 5.8 & 7.2 & 7.3 \\
\hline (E)-a-Bergamotene & 1436 & 1435 & 0.0 & 0.0 & 0.0 & 0.0 & 0.0 & 0.0 & 0.0 & 0.0 & 0.0 & 0.0 & 0.0 & 0.0 & 0.0 & 0.0 & 0.0 & 0.2 & 0.0 & 0.3 \\
\hline$\alpha$-Humulene & 1456 & 1455 & 0.0 & 0.0 & 0.0 & 0.0 & 0.1 & 0.0 & 0.0 & 0.3 & 0.3 & 0.2 & 0.3 & 0.3 & 0.0 & 0.0 & 0.0 & 0.0 & 0.3 & 0.1 \\
\hline$\beta$-Farnesene & 1458 & 1457 & 0.0 & 0.0 & 0.0 & 0.0 & 0.0 & 0.0 & 0.0 & 0.0 & 0.0 & 0.7 & 1.4 & 1.4 & 0.0 & 0.0 & 0.0 & 0.7 & 0.8 & 0.9 \\
\hline Germacrene-D & 1485 & 1485 & 0.0 & 0.0 & 0.0 & 0.0 & 0.0 & 0.0 & 0.3 & 6.9 & 1.2 & 0.6 & 2.8 & 1.3 & 0.7 & 0.8 & 1.2 & 1.5 & 1.2 & 2.2 \\
\hline (E)-Methyl isoeugenol & 1498 & 1492 & 0.0 & 0.0 & 0.0 & 0.0 & 0.0 & 0.0 & 0.0 & 0.0 & 0.0 & 0.2 & 0.0 & 0.1 & 0.0 & 0.0 & 0.0 & 0.0 & 0.0 & 0.0 \\
\hline$\alpha$-Selinene & 1503 & 1498 & 0.0 & 0.0 & 0.0 & 0.0 & 0.0 & 0.0 & 0.0 & 0.0 & 0.0 & 0.0 & 0.0 & 0.0 & 2.1 & 1.9 & 1.3 & 0.0 & 0.0 & 0.0 \\
\hline$\beta$-Bisabolene & 1509 & 1506 & 0.6 & 0.9 & 1.0 & 1.1 & 0.9 & 0.2 & 16.4 & 17.7 & 17.6 & 12.3 & 15.9 & 9.6 & 0.1 & 0.2 & 0.3 & 40.2 & 43.4 & 44.3 \\
\hline$\delta$-Amorphene & 1514 & 1512 & 0.0 & 0.0 & 0.0 & 0.0 & 0.0 & 0.0 & 0.6 & 0.8 & 1.4 & 0.0 & 0.0 & 0.0 & 0.0 & 0.0 & 0.0 & 0.0 & 0.0 & 0.0 \\
\hline$\delta$-Cadinene & 1525 & 1523 & 0.0 & 0.0 & 0.0 & 0.0 & 0.0 & 0.0 & 0.2 & 0.3 & 0.3 & 0.0 & 0.4 & 0.3 & 0.0 & 0.0 & 0.0 & 0.6 & 0.6 & 0.7 \\
\hline$\beta$-Sesquiphellandrene & 1526 & 1523 & 0.0 & 0.0 & 0.0 & 0.0 & 0.0 & 0.0 & 0.0 & 0.0 & 0.0 & 0.0 & 0.7 & 0.5 & 0.0 & 0.0 & 0.0 & 0.0 & 0.0 & 0.0 \\
\hline (E)-Calamenene & 1529 & 1529 & 0.0 & 0.0 & 0.0 & 0.0 & 0.0 & 0.0 & 0.0 & 0.0 & 0.0 & 0.0 & 0.0 & 0.0 & 0.4 & 0.4 & 0.2 & 0.0 & 0.0 & 0.0 \\
\hline$\alpha$-Cadinene & 1539 & 1539 & 0.0 & 0.0 & 0.0 & 0.0 & 0.0 & 0.0 & 0.2 & 0.0 & 0.0 & 0.0 & 0.0 & 0.0 & 0.0 & 0.0 & 0.0 & 0.3 & 0.2 & 0.3 \\
\hline Elemol & 1552 & 1550 & 0.0 & 0.0 & 0.0 & 0.0 & 0.0 & 0.0 & 0.0 & 0.0 & 0.0 & 0.0 & 0.2 & 0.0 & 0.0 & 0.0 & 0.0 & 0.0 & 0.0 & 0.0 \\
\hline Germacrene D-4-ol & 1576 & 1576 & 0.0 & 0.0 & 0.0 & 0.4 & 0.4 & 0.0 & 0.4 & 0.0 & 0.6 & 0.0 & 0.0 & 0.0 & 0.0 & 0.0 & 0.0 & 0.0 & 0.0 & 0.0 \\
\hline Caryophyllene oxyde & 1584 & 1583 & 2.3 & 2.6 & 2.1 & 3.3 & 2.7 & 2.4 & 0.4 & 1.2 & 0.8 & 0.2 & 6.1 & 4.8 & 2.2 & 1.9 & 3.1 & 6.2 & 4.3 & 4.5 \\
\hline$\beta$-Atlantol & 1610 & 1608 & 0.0 & 0.0 & 0.0 & 0.0 & 0.0 & 0.0 & 0.3 & 0.2 & 0.2 & 1.3 & 1.9 & 1.1 & 0.0 & 0.0 & 0.0 & 0.4 & 0.2 & 0.2 \\
\hline Cubenol $<1,10$-di-epi $>$ & 1619 & 1619 & 0.0 & 0.0 & 0.0 & 0.0 & 0.0 & 0.0 & 0.0 & 0.0 & 0.0 & 0.0 & 0.0 & 0.0 & 1.1 & 0.7 & 0.5 & 4.0 & 2.5 & 2.6 \\
\hline Eudesmol $<10$-epi-g $>$ & 1626 & 1624 & 0.0 & 0.0 & 0.0 & 0.0 & 0.0 & 0.0 & 0.4 & 0.2 & 0.4 & 0.0 & 0.0 & 0.0 & 0.0 & 0.0 & 0.0 & 0.4 & 0.1 & 0.1 \\
\hline$\tau$-Cadinol & 1649 & 1646 & 0.1 & 0.0 & 0.1 & 0.1 & 0.0 & 0.2 & 1.4 & 1.7 & 3.3 & 0.6 & 0.6 & 0.3 & 8.0 & 5.1 & 3.9 & 0.1 & 0.2 & 0.3 \\
\hline$\alpha$-Cadinol & 1657 & 1654 & 0.0 & 0.0 & 0.0 & 0.0 & 0.0 & 0.0 & 0.0 & 0.0 & 0.0 & 0.7 & 0.0 & 0.3 & 0.0 & 0.0 & 0.0 & 0.0 & 0.0 & 0.0 \\
\hline Eudesmol <7-epi-a-> & 1668 & 1664 & 0.0 & 0.0 & 0.0 & 0.0 & 0.0 & 0.0 & 0.3 & 0.0 & 0.4 & 0.0 & 0.3 & 0.0 & 0.0 & 0.0 & 0.0 & 0.2 & 0.0 & 0.0 \\
\hline Bisabolol $<$ epi-a-> & 1683 & 1685 & 0.1 & 0.0 & 0.0 & 0.0 & 0.0 & 0.0 & 0.0 & 0.0 & 0.2 & 0.8 & 0.9 & 0.5 & 0.0 & 0.0 & 0.0 & 1.3 & 0.5 & 0.4 \\
\hline Eudesma-4(15),7-dien-1- $b$-ol & 1692 & 1688 & 0.0 & 0.0 & 0.0 & 0.0 & 0.0 & 0.0 & 0.1 & 0.2 & 0.0 & 0.5 & 0.6 & 0.5 & 0.0 & 0.0 & 0.0 & 3.1 & 1.5 & 1.9 \\
\hline Hexadecanoic acid & 1980 & 1960 & 0.0 & 0.0 & 0.0 & 0.0 & 0.0 & 0.0 & 0.0 & 0.0 & 0.0 & 0.0 & 0.0 & 0.0 & 0.0 & 0.0 & 0.0 & 0.5 & 0.1 & 0.1 \\
\hline Monoterpene compounds & & & 93.9 & 92.1 & 93.3 & 87.5 & 89.3 & 92.4 & 74.3 & 64.5 & 70.4 & 71.7 & 49.0 & 62.9 & 79.4 & 84.4 & 82.1 & 28.4 & 30.9 & 25.2 \\
\hline Monoterpene aliphatic hydroc & carbons & & 9.2 & 9.6 & 7.7 & 11.0 & 14.0 & 12.2 & 22.5 & 18.7 & 19.6 & 11.4 & 4.0 & 11.1 & 26.5 & 19.1 & 16.7 & 0.6 & 0.0 & 0.5 \\
\hline Monoterpene aromatic hydroc & carbons & & 60.0 & 58.4 & 67.7 & 35.0 & 36.4 & 57.3 & 15.3 & 25.1 & 20.4 & 15.1 & 7.0 & 18.6 & 18.1 & 36.2 & 48.2 & 1.0 & 0.0 & 0.2 \\
\hline Oxygenated monoterpenes & & & 24.7 & 24.1 & 17.9 & 41.5 & 38.9 & 22.9 & 36.5 & 20.7 & 30.4 & 45.2 & 38 & 33.2 & 34.8 & 29.1 & 17.2 & 26.8 & 30.9 & 24.5 \\
\hline
\end{tabular}


Table 3 (continued)

\begin{tabular}{|c|c|c|c|c|c|c|c|c|c|c|c|c|c|c|c|c|c|c|}
\hline \multirow{3}{*}{$\begin{array}{l}\text { Localities } \\
\text { Oxygenated monoterpenes (no phenolic) }\end{array}$} & \multirow{2}{*}{\multicolumn{3}{|c|}{$\begin{array}{l}\text { Bosnia } 1 \\
\text { (Konnic/ } \\
\text { Spiljani) }\end{array}$}} & \multirow{2}{*}{\multicolumn{3}{|c|}{$\begin{array}{l}\text { Bosnia } 2 \\
\text { (Borci/ } \\
\text { Bjelasnica) }\end{array}$}} & \multirow{2}{*}{\multicolumn{3}{|c|}{$\frac{\text { Sicily }}{\text { (Marettimo) }}$}} & \multirow{2}{*}{\multicolumn{3}{|c|}{$\begin{array}{l}\text { Ibiza } \\
\text { (Balearic } \\
\text { Islands) }\end{array}$}} & \multirow{2}{*}{\multicolumn{3}{|c|}{$\frac{\text { Valencia }}{\text { (Spain) }}$}} & \multirow{2}{*}{\multicolumn{3}{|c|}{$\begin{array}{l}\text { Majorca } \\
\text { (Balearic } \\
\text { Islands) }\end{array}$}} \\
\hline & & & & & & & & & & & & & & & & & & \\
\hline & 3.3 & 4.8 & 3.8 & 3.1 & 2.5 & 4.6 & 10.7 & 4.2 & 4.6 & 2.9 & 1.2 & 2.8 & 15.1 & 9.4 & 14.7 & 0.4 & 0.6 & 1.1 \\
\hline Oxygenated monoterpenes (phenolic) & 21.4 & 19.3 & 14.1 & 38.4 & 36.4 & 18.3 & 25.8 & 16.5 & 25.8 & 42.3 & 36.8 & 30.4 & 19.7 & 19.7 & 2.5 & 26.4 & 30.3 & 23.4 \\
\hline Sesquiterpene compounds & 4.9 & 6.2 & 5.5 & 8.8 & 5.9 & 5.2 & 21.5 & 32.9 & 27.6 & 24.2 & 44.5 & 31.2 & 19.5 & 14.6 & 15.4 & 65.3 & 63.2 & 67.0 \\
\hline Sesquiterpene hydrocarbons & 2.4 & 3.6 & 3.3 & 5.1 & 2.8 & 2.7 & 18.2 & 29.3 & 21.8 & 20.2 & 33.9 & 23.8 & 8.2 & 6.8 & 7.9 & 49.6 & 54.0 & 57.0 \\
\hline Oxygenated sesquiterpenes & 2.5 & 2.6 & 2.2 & 3.7 & 3.1 & 2.5 & 3.3 & 3.6 & 5.8 & 4.0 & 10.6 & 7.4 & 11.3 & 7.8 & 7.5 & 15.7 & 9.2 & 10.0 \\
\hline Others compounds & 1.1 & 1.6 & 0.9 & 2.4 & 3.4 & 1.4 & 2.2 & 1.4 & 0.6 & 1.1 & 0.3 & 1.0 & 0.4 & 0.7 & 0.5 & 0.5 & 0.1 & 0.6 \\
\hline Total & 99.9 & 99.9 & 99.7 & 98.7 & 98.6 & 99.0 & 98.0 & 98.8 & 98.6 & 97.0 & 93.8 & 95.1 & 99.3 & 99.7 & 98.0 & 94.2 & 94.2 & 92.8 \\
\hline
\end{tabular}

a KIE: Kovats (retention) index, experimentally determined, relative to C7-C24 n-alkanes on the ZB-5 column.

b KIL: Kovats (retention) index, literature data (Adams, 2007).

\subsection{Essential oil distribution}

Discriminant analysis (LDA) was performed to develop a model of the distribution of component oils on the basis of locality.

The first two factors explained $97 \%$ of the variability. Prior to this analysis the number of compounds had been reduced based on correlation coefficients $>0.95$. The Majorca populations were found to be highly correlated with positive values of the first factor (F1), whereas the Bosnio-Herzegovinian and Valencia populations were correlated with negative values of this factor. Populations from Ibiza and Sicily were significantly correlated with positive and negative values of the second (F2) factor, respectively (Fig. 3).

In the $\mathrm{F} 1$ discriminant function, $\beta$-bisabolene, carvacrol and germacrene-D were the major compounds differentiating the populations of Majorca, Ibiza and Sicily from those of Bosnia and Valencia. Caryophyllene and $\gamma$-terpinene were the principal compounds differentiating the Ibiza and Sicily populations (function F2). The distribution plot gives four groups characterized by a combination of five monoterpenes and three sesquiterpenes. The compounds $\beta$-bisabolene, carvacrol, $\gamma$-terpinene, $p$ cymene, 1 -terpinen 4 - ol, and camphene were the only components enabling statistically significant $(P<0.05)$ differentiation of the two chemotypes (Table 4).

The chemical distances between the Ibiza and Sicilian populations were principally determined by the presence and concentration of p-cymene, thymol and carvacrol.

\subsection{Ecological factors affecting chemical variability}

Although there was no statistically significant variation in the monoterpenes among the chemotypes, two chemotypes were clearly spatially segregated in relation to a complex of environmental factors (climate and soils). The sesquiterpene chemotype was confined to high altitude areas, in limestone rock crevices having an elevated ombrothermic index value and low values for the thermicity and continentality indices (Tables 1 and 2). In contrast, the aromatic monoterpene chemotype occupied habitats that are wet during summer, and have a high Jacob index and deeper soils. These conditions are also related to the occurrence of non-phenolic chemotypes of Thymus vulgaris L. (Granger and Passet, 1973; Thompson et al., 2003).

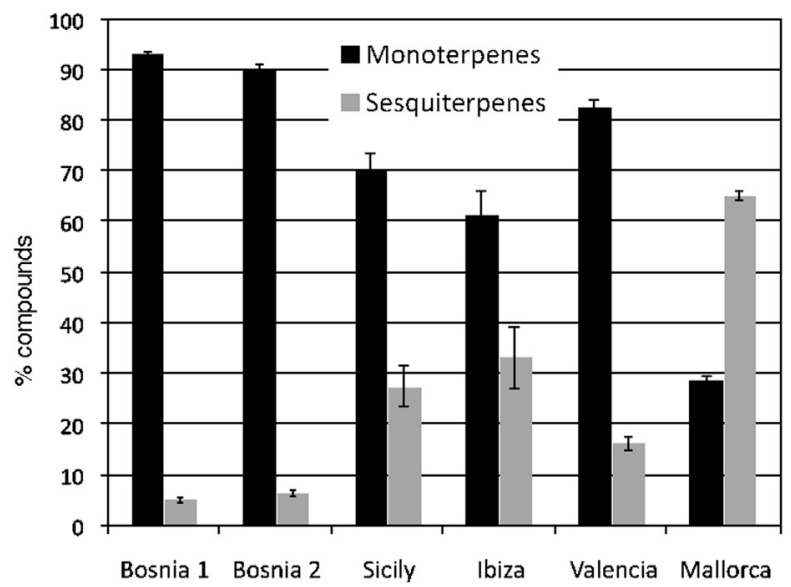

Fig. 2. Variability of two principal components groups of $T$. richardi essential oils (monoterpenes and sesquiterpenes) in the studied populations (mean values, $\% \pm$ s.e). 


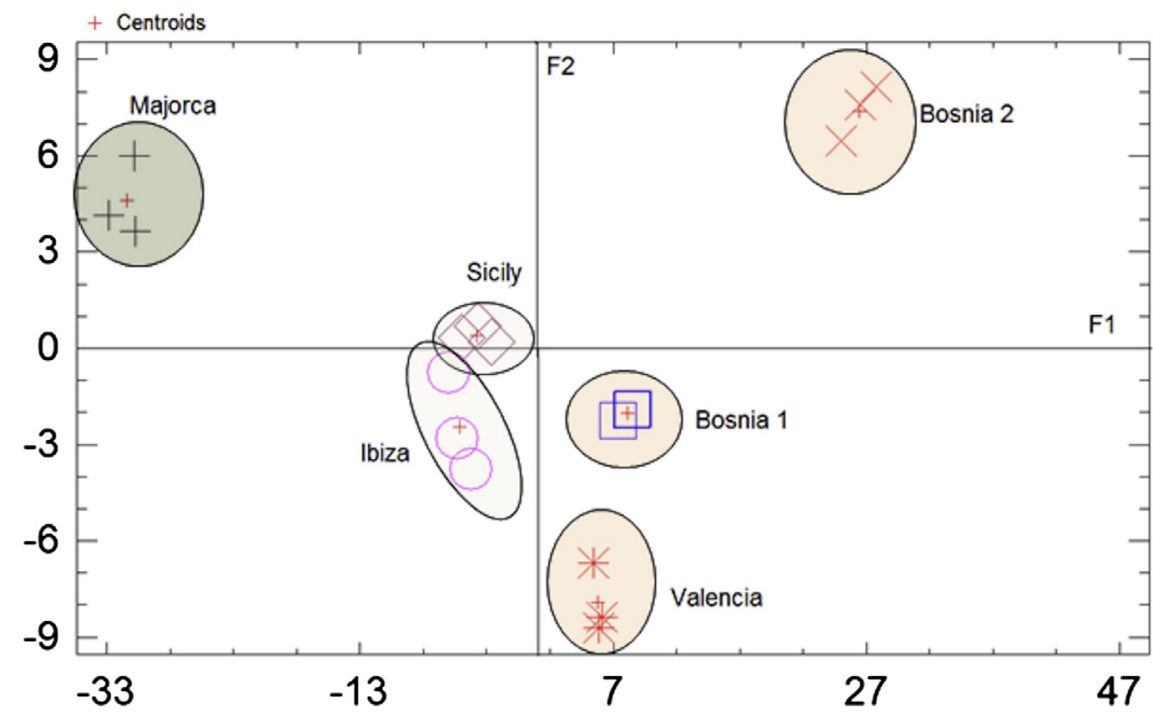

F1 $=5,89^{\star}$ camphene $-4,72^{\star} \gamma \_$terpinene $-5,82^{\star} p_{-}$cymene $-1,85^{\star}$ 1_terpinen_4_ol $+0,23^{\star}$ thymol $-4,14^{\star}$ carvacrol $-2,58^{\star} \beta$ _bisabolene $-3,77^{\star}$ caryophyllene oxyde $1,73^{*} 3$ _octanone.

F2 $=3,67$ camphene $-2,98^{*} \gamma$ _terpinene $-2,80^{*}$ p_cymene $-1,73^{*} 1$ terpinen_4_ol + $0,45^{\star}$ thymol $-2,19^{\star}$ carvacrol $-0,24^{\star} \beta$ _bisabolene $-1,96^{\star}{ }^{\star}$ charyophyllene oxide $0,64^{*} 3$ _octanone.

Fig. 3. Population distributions according the two first discriminant functions. Relative eigenvalue percentage: $F 1=89.77 \% ; F 2=7.31 \%(P<0.01)$.

In terms of practical applications, the most characteristic chemicals in Thymus spp. are the phenolic compounds (mainly thymol and carvacrol). Several studies have investigated environmental influences on their occurrence. Gouyon et al. (1986) pointed out that there is a relatively high correlation between soil type and chemotype structure in $T$. vulgaris, and for this species Thompson et al. (2003) noted earlier studies identifying six chemotypes in southeast France, the occurrence of which were correlated with locations having specific environmental characteristics. The phenolic chemotypes were found in low altitude areas having mild winters and shallow and stony soils, close to the Mediterranean Sea. In contrast, the non-phenolic chemotypes were associated with colder winters and deeper soils. Similar results were reported by Kulevanova et al. (1996) for some Thymus taxa from Macedonia. Samples collected in areas with extreme climatic conditions (hot summers and very cold and snowy winters) correlated with lower levels of phenolic compounds. Gouyon et al. (1986) noted that there was a general trend in relation to the occurrence of phenolic compounds; this indicated that the "home" environment for thyme is stable and xeric in character. This is expressed by the "most chemically elaborate chemotypes, thymol or carvacrol". Similarly, Bruneton (1995) referred to an association between the occurrence of phenolic compounds and typical Mediterranean environments, with non-phenolic chemotypes being correlated with Thymus adapted to different environmental conditions.

Table 4

Major and differential compounds for T. richardii chemotypes: A, p-Cymene; B, $\beta$-Bisabolene.

\begin{tabular}{|c|c|c|c|c|c|c|}
\hline \multirow[b]{2}{*}{ Variable } & \multicolumn{3}{|c|}{ Chemotype A } & \multicolumn{3}{|c|}{ Chemotype B } \\
\hline & Mean & s.e. & $(*)$ & Mean & s.e. & $\left({ }^{*}\right)$ \\
\hline Camphene & 1.17 & 0.21 & $\mathrm{a}$ & 0.00 & 0.00 & $\mathrm{~b}$ \\
\hline$\gamma$-Terpinene & 7.49 & 1.58 & a & 0.00 & 0.00 & $\mathrm{~b}$ \\
\hline p-Cymene & 46.26 & 5.36 & $\mathrm{a}$ & 0.04 & 0.04 & $\mathrm{~b}$ \\
\hline 1-Terpinen-4-ol & 1.47 & 0.43 & a & 0.00 & 0.00 & $\mathrm{~b}$ \\
\hline Thymol & 9.59 & 2.67 & ab & 8.87 & 1.51 & $\mathrm{ab}$ \\
\hline Carvacrol & 0.81 & 0.55 & a & 7.35 & 1.59 & $\mathrm{~b}$ \\
\hline$\beta$-Bisabolene & 0.57 & 0.12 & a & 42.65 & 1.23 & $\mathrm{~b}$ \\
\hline Caryophyllene oxide & 2.50 & 0.15 & $\mathrm{ab}$ & 5.00 & 0.62 & $\mathrm{ab}$ \\
\hline 3-Octanone & 0.38 & 0.17 & $\mathrm{ab}$ & 0.17 & 0.09 & $\mathrm{ab}$ \\
\hline Methyl thymol ether & 8.56 & 2.25 & $\mathrm{ab}$ & 9.72 & 0.88 & $\mathrm{ab}$ \\
\hline
\end{tabular}

Values followed by the same uperscript letter $\left(^{*}\right)$ within the same file are not significantly different. Fisher test of the variance analysis (least significantly difference, LSD; $P<0.05)$.

Bold values signifies major and differential terpenes. 


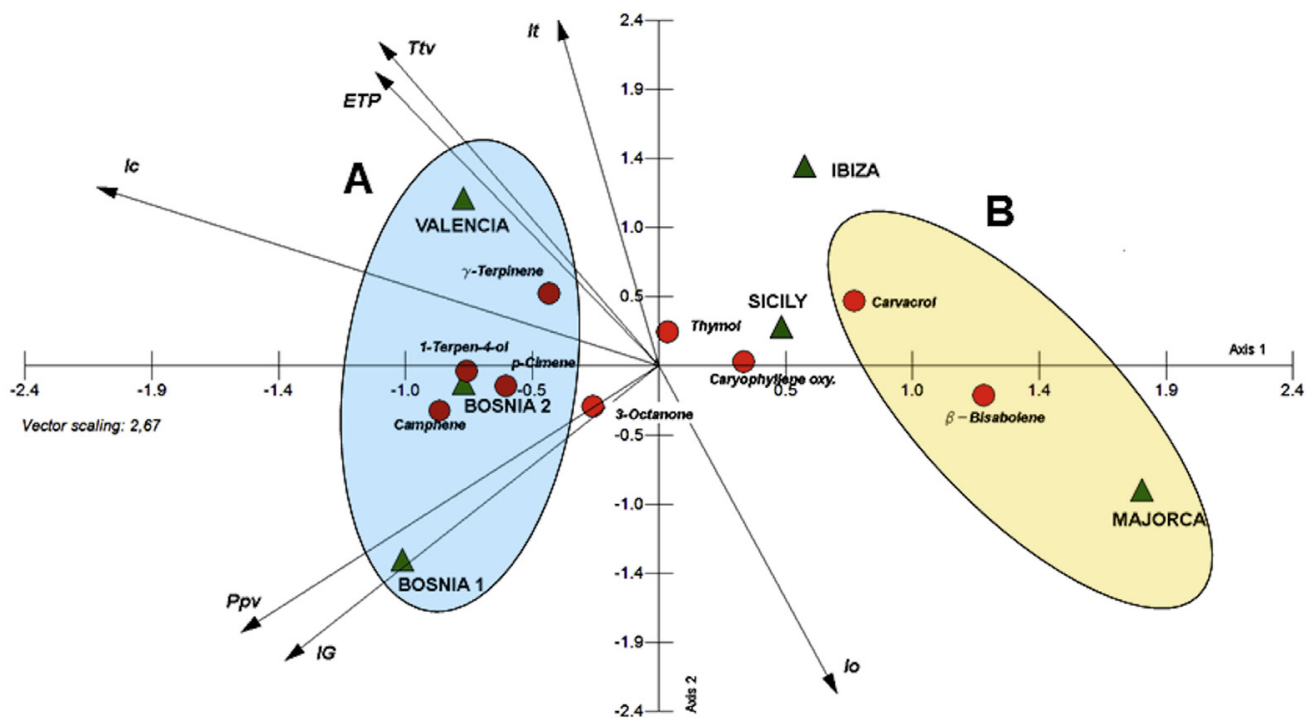

Fig. 4. CCA joint biplot of compounds and localities, and relationships to the bioclimatic indices. A, p-cymene chemotype; B; $\beta$-bisabolene chemotype.

These findings may be correlated with factors other than genetic determinants, as the biosynthetic pathway for terpenoids may change during plant development, under herbivore pressure (Sturgeon, 1979), or because of environmental factors Boira and Blanquer, 1998; Delazar et al., 2011; Robles and Garzino, 2000).

Having little and bimodal rainfall, and subtropical dry summers characterize western and central Mediterranean climates. However, because of geomorphological conditions, variability and interrelations among climatic factors can determine the climatic conditions in particular regional and local (microclimate) locations.

Quantitative differences between chemotypes in the studied populations were related to soil and bioclimatic factors (temperature and water).

The Bosnian populations (Konjic and Borci) were characterized by growing in a temperate or humid Mediterranean microclimate, especially during summer. The essential oils of these populations were related to Valencia populations (Safor) by the high content of aromatic monoterpenes ( $p$-cymene). The Konjic, Borci and Valencia areas have a higher average rainfall than those of the other studied populations. The Safor population (750 $\mathrm{m}$ asl) occurs on a small island $\left(4 \mathrm{~km}^{2}\right) \mathrm{where} \mathrm{ke}$ rainfall reaches $900 \mathrm{~mm}$ annually, and is surrounded by a vast area where the rainfall does not exceed $550 \mathrm{~mm}$. The presence of significant amounts of $\gamma$-terpinene, a differential compound for chemotype A (Valencia and Bosnia), was positively related to ETP, Ttv and IG (availability of water in summer months) (Fig. 4).

The occurrence of $\beta$-bisabolene, germacrene-D and carvacrol (compounds characteristic of chemotype B) was related to positive values of Io. These conditions are common in Ibiza and Sicily, and these parameters reach higher levels in Majorca.

The presence of the phenol chemotypes ( $\gamma$-terpinene, p-cymene, camphene and 1-terpinene-4-ol) in Thymus spp. occurring outside areas having xeric climate conditions seems to be related to the chemical characteristics of the soil (Martonfi et al., 1994).

The populations of Majorca, Ibiza and Marettimo grow in limestone dolomitic rocks, in crevices and cracks on almost vertical rock surfaces (chasmophytes). The Konjic, Borci and Safor biotypes occur on incipient soils (soils characterized by stony rendosol with a poorly developed B horizon) on gentle slopes, and are closely associated with dolomitic bedrock in initial stages of soil development (Table 1).

The data for the volatile components of the various Thymus richardii populations analyzed showed that although most were phenol precursors related to the monoterpenoid fraction, the main differences involved the sesquiterpenoid fraction, with $\beta$-bisabolene being the most representative compound for this species.

The adaptation of the Majorca population to extreme climatic and edaphic conditions of the mountain of Puig Major wasn't associated with a decrease in the amount of phenolic compounds or an increase in non-phenolic monoterpenoids. In this case we found a major increase in the sesquiterpenoid fraction (mainly $\beta$-bisabolene).

Comparison of the more xeric populations and those from an environment having a damper climate and deeper soils (Safor, Konjic and Borci) indicated two trends: (a) precursors of phenolic compounds predominated in Safor, Konjic and Borci samples, whereas the phenolic compounds formed from the precursors were evident to a greater extent in the other wild populations; (b) the Konjic, Borci and Safor (mainly) populations had greater amounts of non-phenolic monoterpenoids. In relation to the distribution of chemotypes of $T$. vulgaris described by Thompson et al. (2003), both trends are consistent with previous reports (Bruneton, 1995; Kukelanova et al., 1996) of the relationship between typical Mediterranean environments and the occurrence of phenolic compounds. 


\section{Acknowledgements}

\section{The author's wish to thank Drs. Jaime Primo, Sandra Vacas (ITQ/CSIC-UPV) and Trinitat García (SCT/UIB) for their help with analytical methods.}

\section{References}

Adams, R.P., 2007. Identification of Essential Oil Components by Gas Chromatography/Mass Spectrometry, fourth ed., Allured Publishing Corporation. Aziz, E.E., Hendawi, S.F., Ezz El-Din, A., Omer, E.A., 2008. Effect of soil type and irrigation intervals on plant growth, essential oil yield and constituents of Thymus vulgaris plant. Am. Euras. J. Agric. Environ. Sci. 4, 443-450.

Bader, A., Flamini, G., Cioni, P.L., Morelli, I., 2001. Thymus richardii Pers. subsp. nitidus (Guss.) Jalas: a new thymus type rich in beta-bisabolene. J. Essent. Oil Res. 13, 8-10.

Blanco, J., Ruiz, T., Vazquez, F.M., Pérez, J., Cases, M.A., 2012. The essential oil of the protected species: Thymus praecox ssp. penyalarensis. Acta Soc. Bot. Pol. $81,23-27$.

Boira, H., Blanquer, A., 1998. Environmental factors affecting chemical variability of essential oils in Thymus piperella L. Biochem. Syst. Ecol. 26, 811-822.

Bolòs, O., Vigo, J., 1983. Notes sobre taxonomia i nomenclatura de plantes, II. Collect. Bot. 14, 89-103.

Bruneton, J., 1995. Elementos de Fitoquímica y de Farmacognosia. Acribia, Zaragoza.

Cavara, S., Maksimović, M., Vidic, D., 2009. The essential oil of Thymus aureopunctatus (Beck) K. Malý. Nat. Prod. Commun. 4, 415-420.

Chaintreau, A., 2001. Simultaneous distillation-extraction: from birth to maturity—review. Flavour Fragr. J. 16, 136-148.

Crocoll, C., Asbach, J., Novak, J., Gershenzon, J., Degenhardt, J., 2010. Terpene synthases of oregano (Origanum vulgare L.) and their roles in the pathway and regulation of terpene biosynthesis. Plant Mol. Biol. 73, 587-603.

Delazar, A., Bahmani, M., Hekmat-Shoar, H., Tabatabaei-Raisi, A., Asnaashari, S., Nahar, L., Sarker, S.D., 2011. Effect of altitude, temperature and soil on essential oil production in Thymus fedtschenkoi flowers in Osko and surrounding areas in Iran. J. Essent. Oil Bear. Pl. 14, 23-29.

Font Quer, P., 1935. De flora occidentale adnotationes, 7, pp. 71-83. XII. Cavanillesia.

Giacobbe, A., 1938. Schema di una teoria ecologica per la classificazione della vegetatione italiana. Nuovo Giorn. Bot. Ital. 45, 37-121.

Giacobbe, A., 1959. Nouvelles recherces écologiques sur l'aridité sans les pays de la Méditerranée occidentale. Nat. Monspel. S. Bot. 11, 7-27.

Giuliani, C., Maleci Bini, L., 2008. Insight into the structure and chemistry of glandular trichomes of Labiatae, with emphasis on subfamily Lamioideae. Plant Syst. Evol. 276 (3-4), 199-208.

Gouyon, P.H., Vernett, Ph, Guillerm, J.L., Valdeyron, G., 1986. Polymorphisms and environment the adaptative value of the oil. Polymorphisms in Thymus vulgaris. Heredity 57, 59-66.

Granger, R., Passet, L., 1973. Thymus vulgaris L. spontané de France: races chimiques et chemotaxonomie. Phytochemistry 12, $1683-1691$.

Gussone, G., 1843. Florae Siculae Synopsis. Typis Tramater. Neapoli vol. 2, 97.

Jalas, J., 1971. Notes on Thymus (Labiatae) in Europe. II. Comments on species and subspecies. J. Bot. J. Linn. Soc. 64, 247-271.

Jalas, J., 1972. Thymus L. In: Tutin, T.G., et al. (Eds.), Flora Europaea, 3. Cambridge University Press, Cambridge, pp. 172-182.

Jassbi, A.R., Asadollahi, M., Masroor, M., Schuman, M.C., Mehdizadeh, Z., Soleimani, M., Miri, R., 2012. Chemical classification of the essential oils of the Iranian Salvia species in comparison with their botanical taxonomy. Chem. Biodivers. 9, 1254-1271.

Kulevanova, S., Ristic, M., Stafilov, T., Dorevski, K., Ristov, T., 1996. Essential oil analysis of some taxa of genera Thymus L. - environment influences-. Bull. Chem. Technol. Maced. 1996 (15), 33-38.

Lakusic, D.V., Ristic, M.S., Slavkovskac, V.N., Jasmina, B., Sinzar-Sekulic, J.B., Lakusic, B.S., 2012. Environment-Related variations of the composition of the essential oils of rosemary (Rosmarinus officinalis L.) in the Balkan peninsula. Chem. Biodivers. 9, 1286-1302.

Letchamo, W., Gosselin, A., 1995. Effects of HPS supplemental lighting and soil water levels on growth, essential oil content and composition of two thyme (Thymus vulgaris L.) clonal selections. Can. J. Plant Sci. 75, 231-238.

Loziene, K., Venskutonis, P.R., 2005. Influence of environmental and genetic factors on the stability of essential oil composition of Thymus pulegioides. Biochem. Syst. Ecol. 33, 517-525.

Martonfi, P., Grejtovsky, A., Repcak, M., 1994. Chemotype pattern differentiation of Thymus pulegioides on different substrates. Biochem. Syst. Ecol. 22, $819-825$.

Morales, R., 1997. Synopsis of the genus Thymus L. in the Mediterranean area. Lagascalia 19, 249-262.

Paaver, U., Orav, A., Arak, E., Maeorg, U., Raal, A., 2008. Phytochemical analysis of the essential oil of Thymus serpyllum L. growing wild in Estonia. Nat. Prod. Res. 22, 108-115.

Persoon, C.H., 1806. Synopsis Plantarum seu Erchidium botanicum complectens Enumerationem systematicam specierum hucusque cognitarum. Paris and Tuebingae.

Pluhar, Z., Hethelyi, E., Kutta, G., Kamondy, L., 2007. Evaluation of environmental factors influencing essential oil quality of Thymus pannonicus All., and Thymus praecox Opiz. J. Herbs. Spices Med. Plants 13, 23-43.

Ritter-Studnička, H., 1967. Reliktgesellschaften auf Dolomitböden in Bosnien und der Hercegovina. Vegetatio 15, 190-212.

Rivas-Martínez, S., Fernández-González, F., Loidi, J., Lousã, M., Penas, A., 2001. Syntaxonomical checklist of vascular plant communities of Spain and Portugal to association level. Itinera Geobot. 14, 5-341.

Rivas-Martínez, S., Rivas Sáenz, S., Penas, A., 2011. Global Geobotany, 1, p. 638. http://dx.doi.org/10.5616/gg110001.

Robles, C., Garzino, S., 2000. Infraspecific variability in the essential oil composition of Cistus monspeliensis leaves. Phytochemistry 53, 71-75.

Sáez, F., Stahl-Biskup, E., 2002a. Thyme. Taylor \& Francis, New York, 331 pp.

Sáez, F., Stahl-Biskup, E., 2002b. Essential oil polymorphism in the genus Thymus. In: Stahl-Biskup, E., Sáez, F. (Eds.), Thyme. Taylor \& Francis, New York, pp. $125-143$.

Sharafzadeh, S., Alizadeh, O., Vakili, M., 2011. Effect of nitrogen sources and levels on essential oil components of Thymus vulgaris L. Aust. J. Basic Appl. Sci. 5, $885-889$.

Skoula, M., Harborne, J.B., 2002. The taxonomy and chemistry of Origanum. In: Kintzios, S.E. (Ed.), Oregano: the Genera Origanum and Lippia. Taylor and Francis, London, pp. 67-108.

Stahl-Biskup, E., 2002. Essential oil chemistry of the genus Thymus - a global view. In: Stahl-Biskup, E., Sáez, F. (Eds.), Thyme. Taylor \& Francis, New York, pp. 75-124.

Sturgeon, K.B., 1979. Monoterpene variation in ponderosa pine xylem resin related to western pine beetle predation. Evolution 33, $803-814$.

Thompson, J.D., 2002. Population structure and the spatial dynamics of genetic polymorphism in thyme. In: Stahl-Biskup, E., Sáez, F. (Eds.), Thyme. Taylor \& Francis, New York, pp. 44-74.

Thompson, J.D., Chalchat, J.C., Michet, A., Linhart, Y.B., Ehrls, B., 2003. Qualitative and quantitative variation in monoterpene co-occurrence and composition in the essential oil of Thymus vulgaris chemotypes. J. Chem. Ecol. 29, 859.

Tzakou, O., Constantinidis, T., 2005. Chemotaxonomic significance of volatile compounds in Thymus samius and its related species Thymus atticus and Thymus parnassicus. Biochem. Syst. Ecol. 33, 1131-1140. 